\title{
Object shape classification by ultrasound for the purpose of car parking assistance system
}

\author{
Masaki Okugumo a,* \\ ${ }^{a}$ National Institute of Technology, Yonago College, 4448 Hikona-cho, Yonago, 683-8502, Japan \\ *Corresponding Author:okugumo@yonago-k.ac.jp
}

\begin{abstract}
Ultrasound sensor systems have characteristics such as small size and easy system configuration, and are applied in various fields such as robots, automobiles, and medical care. In this paper, frequency-modulated ultrasonic waves are emitted to improve the ability to recognize the surface shape of an object, and frequency analysis is performed on the reflected wave from the object using wavelet transform. Based on the intensity of each frequency band extracted from the reflected wave, shape classification is performed using a self-organizing map (SOM).
\end{abstract}

Keywords: ultrasound, shape classification, SOM.

\section{Introduction}

In recent years, with the remarkable development of computer and sensor technology, driving assistance and automation technology such as automatic driving technology, automatic braking system, and parking assist system are attracting attention and are being put into practical use in the automobile industry. Especially in the field of automobiles, application to distance measurement (back sonar) and vehicle height sensor at a relatively short distance before and after the vehicle is known. However, ultrasound sensor systems that are currently in practical use are limited to those that calculate the distance to an object based on the reflection time of the ultrasound waves ${ }^{(1,2)}$.

\section{Shape classification by ultrasound}

The ultrasound sensor system is capable of measuring distances at a low cost and with relatively high accuracy due to the characteristic of slow propagation speed of the sound wave. However, in the ultrasound sensor system generally used, the emitted ultrasound wave is the target object. It is limited to those that measure the distance to the target object based on the reflection time until it returns to the sensor system. Therefore, the development of a highly functional ultrasound sensor system is desired for the purpose of making the ultrasound sensor system currently used only for simple applications compatible with advanced driving assistance such as parking assistance. Therefore, in this paper, we conducted basic research on an ultrasound sensor system that roughly categorizes the shapes of target objects by radiating frequency-modulated ultrasound waves and using frequency analysis of the reflected waves and a self-organizing map (SOM).

\section{Proposed ultrasound sensor system}

Figure 1 shows the structure of the sensor system. This sensor system can be broadly divided into three processing units: frequency-modulated ultrasound radiation, frequency analysis of reflected waves, and shape classification by a self-organizing map (SOM). The processing of each part is explained below.

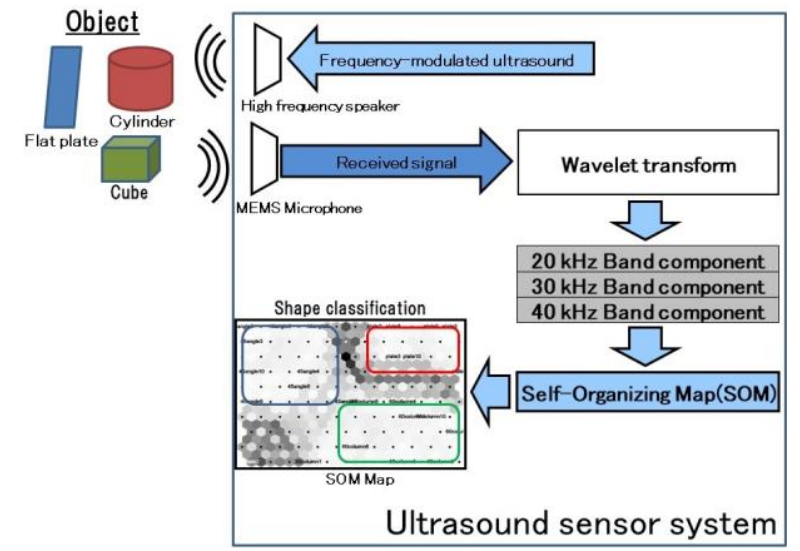

Fig. 1. Ultrasound sensor system. 


\subsection{Frequency modulated ultrasound}

The proposed ultrasound sensor system uses a single-frequency ultrasound wave in the general ultrasound sensor system, and the frequency is continuously changed by decreasing the frequency by $2 \mathrm{kHz}$ every time one wavelength is output from $40 \mathrm{kHz}$ to $20 \mathrm{kHz}$. It emits ultrasound waves with the characteristics of going. As a result, measurement data can be analyzed in a wide frequency band, which is considered effective for object shape recognition ${ }^{(3)}$.

\subsection{Frequency analysis of received wave}

Wavelet transform is one of the time-frequency analysis methods that simultaneously extract the signal from both the time and frequency by extracting only the part similar to the waveform called mother wavelet from the signal to be analyzed $^{(4,5)}$.

The wavelet transform signal $\mathrm{W}(\mathrm{b}, \mathrm{a})$ of the signal $\mathrm{x}(\mathrm{t})$ is defined as the following equation (1).

$$
\mathrm{W}(\mathrm{b}, \mathrm{a})=\frac{1}{\sqrt{\mathrm{a}}} \int_{-\infty}^{\infty} \mathrm{x}(\mathrm{t}) \bar{\varphi}\left(\frac{\mathrm{t}-\mathrm{b}}{\mathrm{a}}\right) \mathrm{dt}
$$

Where $\varphi(t)$ is a transformation function called mother wavelet. $W(b, a)$ is obtained by this equation indicates the correlation between the signal $\mathrm{x}(\mathrm{t})$ and the mother wavelet $\varphi(t)$, and is called the continuous wavelet transform of $x(t)$. Parameter "a" is a variable called scale parameter, and parameter " $b$ " is a variable called shift parameter.

In the wavelet transform, in order to partially extract the signal of interest, $t$ in the mother wavelet $\varphi(t)$ is replaced with "(t-b)/a" using the scale parameter "a" and shift parameter $b$.

The scale parameter " $a$ " is a positive real number used to determine the expansion ratio of the mother wavelet. The low frequency (high scale) corresponds to rough information of the signal, while the high frequency (low scale) is hidden in the signal. It corresponds to the detailed information of the pattern.

Also, the shift parameter " $b$ " is a real number that determines the shift amount of the mother wavelet in the time direction.

In this paper, we used Gabor wavelet, which is effective for analyzing temporally localized periodic waveforms, as frequency analysis of received waves. The definition of Gabor wavelet is given by the following equation (2).

$$
\varphi(t)=\frac{1}{\sqrt{2 \pi \sigma^{2}}} e^{-\frac{t^{2}}{2 \sigma^{2}}} e^{j \omega t}
$$

In equation (2), $\sigma$ is a constant. If this is large, the frequency distribution will be accurate, and if it is small, the temporal change will be accurate.

In the wavelet transform, it is important to obtain a scale parameter according to the frequency to be analyzed. In this paper, the reflected wave sampling speed is $200 \mathrm{ksps}$, and when the frequency of the reflected wave itself is 20 $\mathrm{kHz}, 10$ samples is one cycle. Therefore, the mother wavelet is made so that there is 10 samples and one cycle, and when the scale parameter is 1 , the mother wavelet is adjusted to correspond to $20 \mathrm{kHz}$. Similarly, when measuring the frequency component of $30 \mathrm{kHz}$, the scale parameter " $a$ " is set to 0.666 , and when measuring the frequency component of $40 \mathrm{kHz}$, the mother wavelet is reduced. The analysis results of 200 points are obtained while shifting the 2048 received waveforms sampled as described above at intervals of 10 points.

\subsection{Classification of object shapes by SOM}

Self-organizing map (SOM) is a kind of unsupervised learning neural network, and is an effective technique for classification and analysis of multidimensional data published by T. Kohonen around $1981^{(6)}$. By learning an n-dimensional vector group, the relationship can be shown on a two-dimensional map. Approximated vectors are arranged at close positions on the map, and non-approximate vectors are distant positions. Is placed at. The big feature is that the classification and similarity of data can be visualized. Figure 2 shows the concept of shape classification using SOM and an example of SOM.

As data to be input to the SOM, frequency-modulated ultrasonic waves are emitted to the target object, and several points before and after the reflected wave arrival time are extracted to the SOM in the time-frequency spectrum extracted in the 20,30,40 KHz frequency band by wavelet transform. Used as the input dimension number for. The data extracted by the wavelet transform is normalized and extracted at $20 \mathrm{kHz}, 30 \mathrm{kHz}$, and $40 \mathrm{kHz}$. Therefore, 10 points for the $20 \mathrm{kHz}$ component data, 15 points for the $30 \mathrm{kHz}$ component data, and 20 points for the $40 \mathrm{kHz}$ component data. Let the reflected wave component of the data to be converted to SOM be constant. 


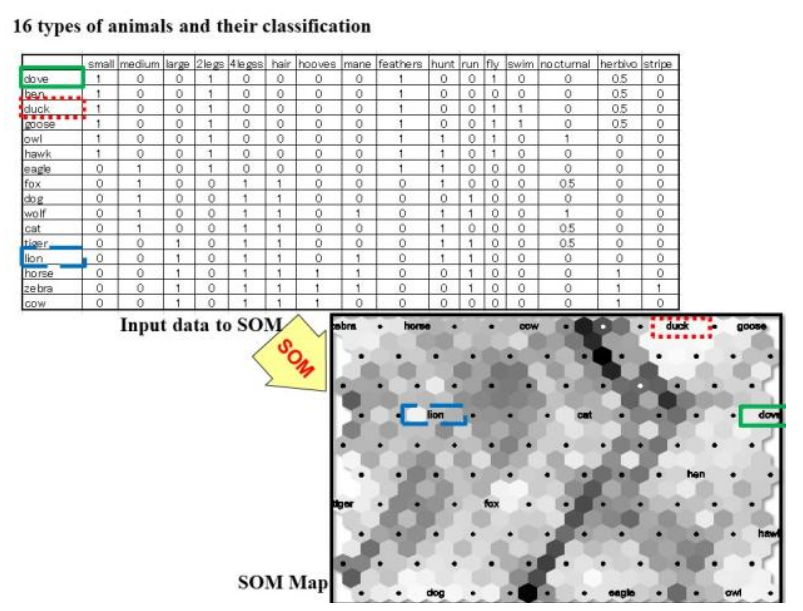

Fig. 2. Self-organizing map (SOM).

\subsection{System configuration}

Figure 3 shows the hardware configuration of the ultrasonic sensor system.

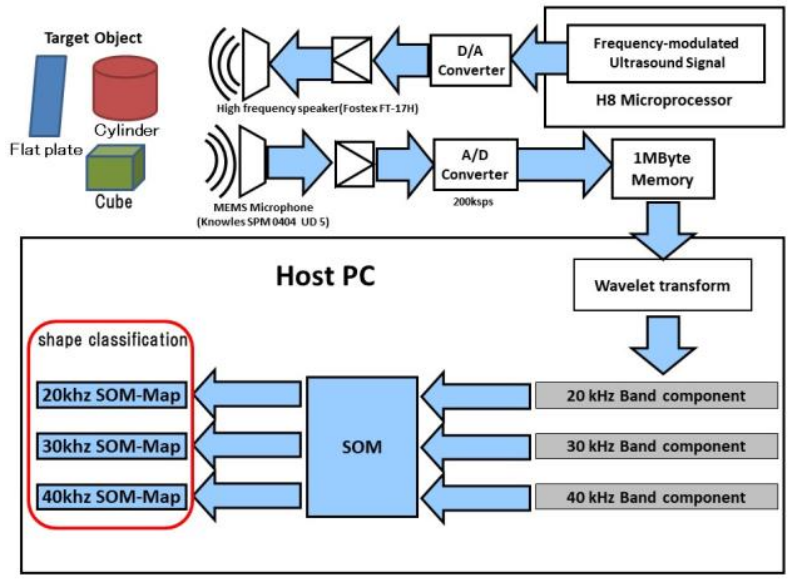

Fig. 3. System configuration.

A high-frequency speaker is used for ultrasound transmission and a broadband microphone is used for reception. The basic hardware control is performed by a 16-bit one-chip microcomputer (H8), and the received wave is processed by a Host PC. The frequency modulated ultrasound waveform is recorded as digital data in the built-in ROM of the sub CPU. The operation of the system is shown below.

(1) $\mathrm{D} / \mathrm{A}$ conversion based on the waveform data stored in the sub CPU to generate and amplify the transmission ultrasound wave, and then output from the speaker.

(2) The reflected wave is received by a MEMS microphone, amplified, and stored in a RAM of about 10 msec (2048 samples) at a sampling frequency of $200 \mathrm{kHz}$ using an $\mathrm{A} / \mathrm{D}$ converter.

(3) Transfer the reflected waveform stored in RAM to the HOST PC via USB.

(4) Perform signal processing and SOM generation in the HOST PC.

\section{Shape classification experiment}

As an example of obstacles that exist in the vicinity of a car garage or parking, three types of objects with a $190 \mathrm{~mm} \times 135 \mathrm{~mm}$ flat plate, a $60 \mathrm{~mm}$ diameter cylinder, and a cube with a side of $70 \mathrm{~mm}$ inclined at $45^{\circ}$ are each $23 \mathrm{~cm}$ in front of the sensor system. The shape classification method was evaluated by installing it at the position of. The measurement is performed 10 times for each target object, and time frequency data of 20,30 , and $40 \mathrm{kHz}$ is extracted using wavelet transform. Several points before and after the reflected wave arrival time of these data are used as the dimension number of SOM. As an example, figure 4 shows sample points as dimensions used for SOM of wavelet analysis data of a flat plate.

Since the created data is data normalized by extracting the intensity at $20 \mathrm{kHz}, 30 \mathrm{kHz}$, and $40 \mathrm{kHz}$, respectively, the same as the cross-correlation coefficient, 10 points before and after $20 \mathrm{kHz}$ component data, 15 points before and after $30 \mathrm{kHz}$ component data, and $40 \mathrm{kHz}$ component data Acquire 20 points before and after, and make the reflected wave component of the data to be SOM constant. The learning parameters for SOM are shown below.

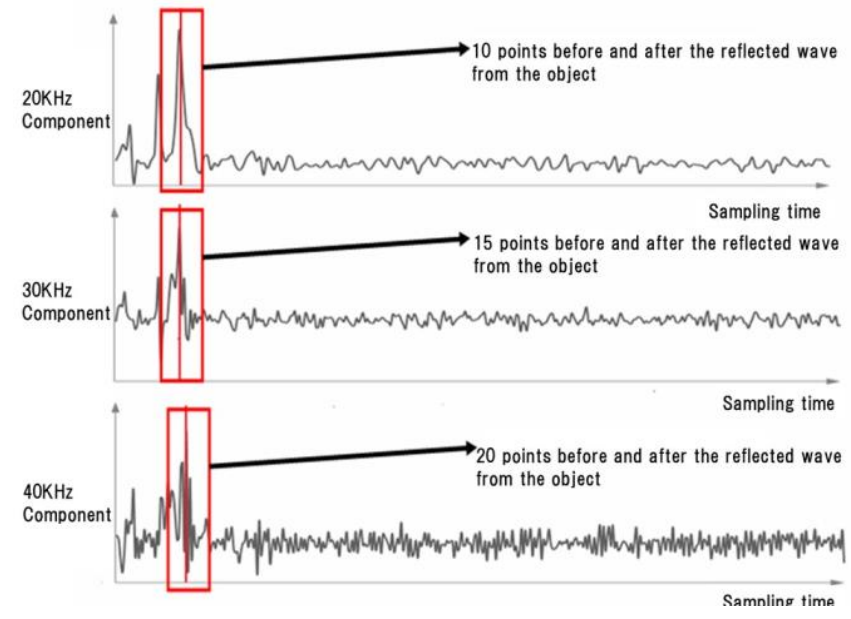

Fig. 4. Input data to SOM. 
Table 1. Learning parameters for SOM.

\begin{tabular}{|l|l|}
\hline Map Size & $12 \times 10$ \\
\hline Learning Coefficient & 0.04 \\
\hline Neighborhood Radius & 30 \\
\hline Learning Times & 7000 \\
\hline Random Coefficient & 30 \\
\hline Gaussian function threshold & 0.03 \\
\hline
\end{tabular}

\section{Result and discussion}

Figure 5-7 shows the SOM for each of the $20 \mathrm{kHz}, 30$ $\mathrm{kHz}$, and $40 \mathrm{kHz}$ components using the data measured 10 times for each of three types: a flat plate, a cylinder, and a cube. It shows where the data for a single cylinder with a diameter of $36 \mathrm{~mm}$ are classified as obstacle data.

And, Fig. 8-10 shows where the data for a single angle of $300^{\circ}$ on one side and a $30^{\circ}$ tilt angle is classified as the actual obstacle data in the same SOM. Here, labels are labeled as follows: plate1-10 is 1-10th measurement on a flat plate, 60column1-10 is measurement 1-10th measurement on a $60 \mathrm{~mm}$ diameter cylinder, 45angle $1-10$ is measurement at a cube with a side of $70 \mathrm{~mm}$ inclined at $45^{\circ}$ are each $23 \mathrm{~cm}$. And 30 anglelis indicates the first measurement at a corner with angle inclined by $30^{\circ}$.

A hexagon-delimited area on the SOM is called a node. If this node is white, it indicates that it is highly related to neighboring nodes, and if it is black, it indicates that the relationship is low. From these results, in each frequency band, the labels of the flat plate, cylinder, and corner are gathered relatively close on the SOM, and there is a black node at the boundary of the set of labels for each object shape. This shows that the area is classified by each shape.

Moreover, since black lines are generated in the flat region and the cylinder region, and in the flat plate region and the cube region, the reflected wave analysis data of the flat plate and the cylinder, and the flat plate and the cube are less relevant. However, gray lines are mainly generated in the cylinder region and the corner region, so it is considered that the relationship between the cylinder and the corner is higher than that of the flat plate and the cylinder node. This is thought to be related to the fact that the reflectivity of ultrasound waves varies depending on the object shape, and that the flat plate has the highest reflectivity, so it is reflected with a higher intensity than a cylinder or corner. The analysis data of the reflected wave of the cylinder and the corner are more relevant because these frequency spectrum intensities are close.
Furthermore, as a result of classification of $36 \mathrm{~mm}$ diameter cylinders measured as actual obstacle data, as shown in figure 5 , the $36 \mathrm{~mm}$ diameter cylinder measurement data is close to the area of the $60 \mathrm{~mm}$ diameter cylinder node in the SOM frequency bands of 20 $\mathrm{kHz}$. From the position, it can be seen that the shape is similar to a cylinder. However, the SOM with a frequency of $30 \mathrm{kHz}$ and $40 \mathrm{kHz}$ shown in figure 6 and 7 is located near the boundary between the corner and the plate, and the classification is ambiguous in this frequency of $3040 \mathrm{kHz}$.

From Figure 8-10, it can be seen that the measurement node with a $30^{\circ}$ corner shows a shape similar to that of the corner by being close to the area of the cube node in any frequency band. Classification is considered to be relatively common.From these results, it is considered that there is a frequency band suitable for the shape of the obstacle, and it can be said that the classification method using multiple frequency bands is more effective than the classification by single frequency. Moreover, it can be seen that the measurement data of target objects with similar classification shapes are approximate, and the possibility of classification of target object shapes can be confirmed.

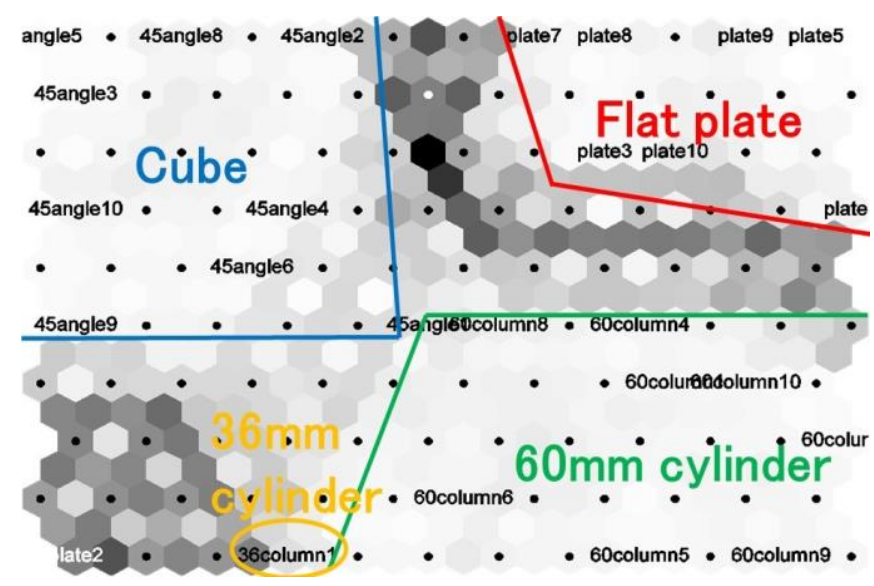

Fig. 5. $20 \mathrm{kHz}$ SOM-Map with 36mm cylinder.

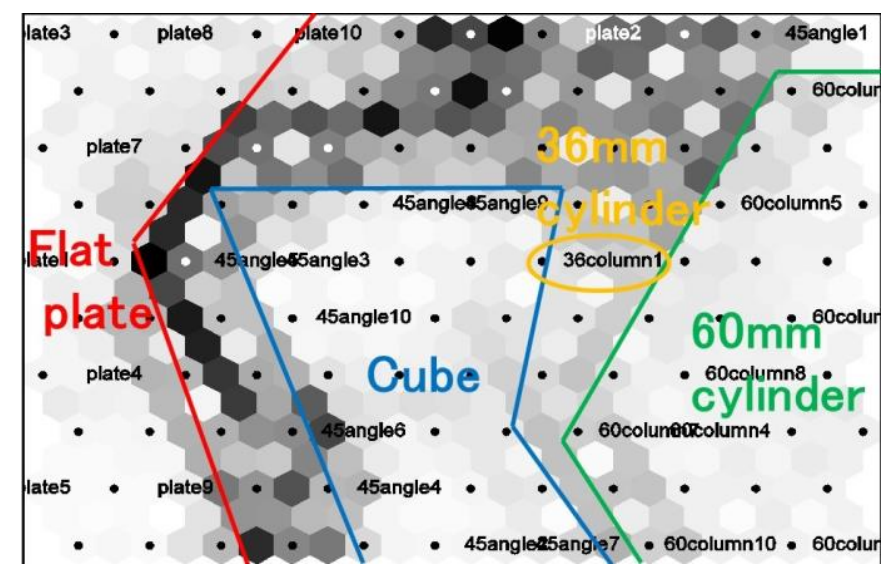

Fig. 6. $30 \mathrm{kHz}$ SOM-Map with $36 \mathrm{~mm}$ cylinder 


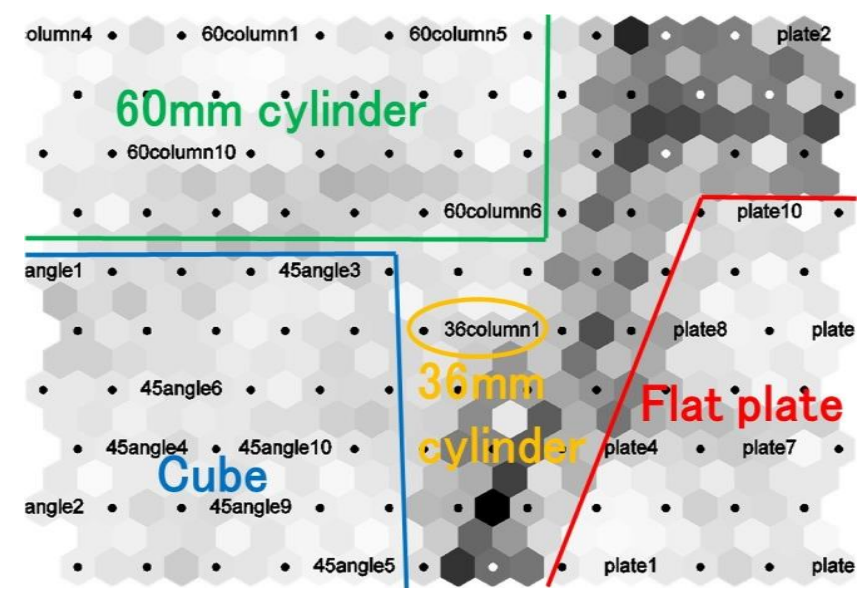

Fig.7. $40 \mathrm{kHz}$ SOM-Map with $36 \mathrm{~mm}$ cylinder.

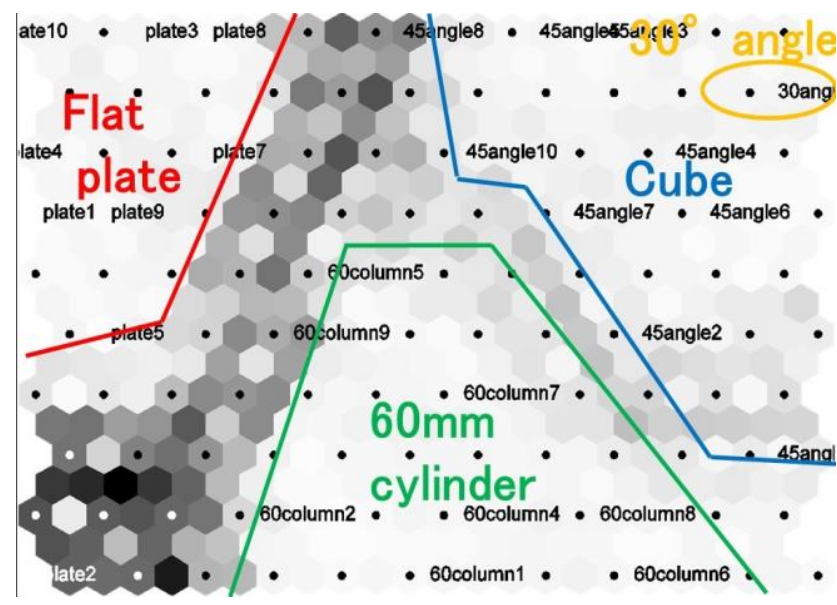

Fig.8. $20 \mathrm{kHz}$ SOM-Map with $30^{\circ}$ angle.

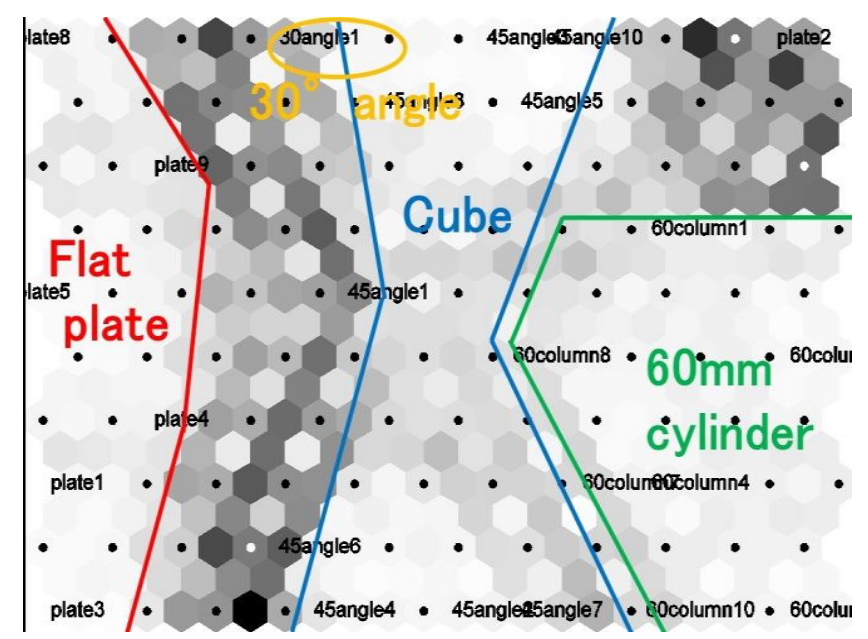

Fig.9. $\quad 30 \mathrm{kHz}$ SOM-Map with $30^{\circ}$ angle.

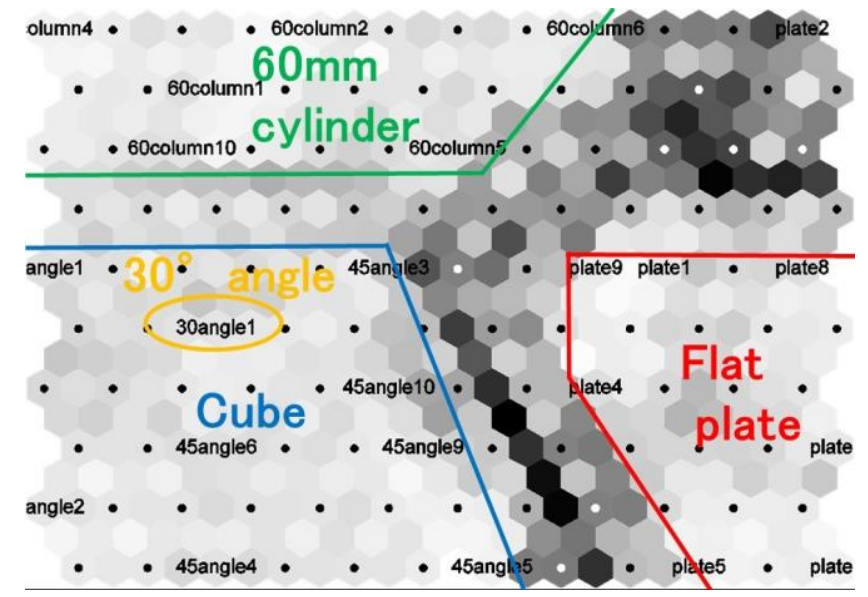

Fig.10. $40 \mathrm{kHz}$ SOM-Map with $30^{\circ}$ angle

\section{Conclusions}

In this paper, we described the possibility of an ultrasonic sensor system for a parking assistance system using frequency-modulated ultrasound and self-organizing map (SOM). This method roughly classifies and visualizes the shape of the target object by using frequency-modulated ultrasound, frequency analysis by wavelet transform, and SOM as a neural network. In the future, it will be necessary to improve the classification accuracy of the target object and verify it in the vehicle environment.

\section{References}

(1) Stefan Kocis, and Zdenko Figura : "Ultrasonic Measurements and Technologies: Engineering Applications (Sensor Physics and Techniques Series Book 4)", Springer, 2012

(2) S. Arefin, and T. Mollick : "Design of an Ultrasonic Distance Meter", International Journal of Scientific \& Engineering Research, Vol.4, pp. 1-10, 2013

(3) M. Okugumo, A. Kimura, M. Ohki, and M. Ohkita : "Development research on high performance ultrasound sensor system", Electronics and Communications in Japan, , No.92, pp. 23-30, 2009

(4) Ingrid Daubechies : "Ten Lectures on Wavelets", Society for Industrial and Applied Mathematics, 1992

(5) Guy Nason : "Wavelet Methods in Statistics with R", Springer, 2008

(6) Teuvo Kohonen : "Self-Organizing Maps", Springer Verlag, 1995 